\title{
A foot/ground contact model for biomechanical inverse dynamics analysis
}

\author{
Romain Van Hulle*, Cédric Schwartz*, Vincent Denoël*, Jean-Louis Croisier*, Bénédicte Forthomme*, Olivier Brüls* \\ Laboratory of Human Motion Analysis, University of Liège, Belgium
}

\begin{abstract}
The inverse dynamics simulation of the musculoskeletal system is a common method to understand and analyse human motion. The ground reaction forces can be accurately estimated by experimental measurements using force platforms. However, the number of steps is limited by the number of force platforms available in the laboratory. Several numerical methods have been proposed to estimate the ground reaction forces without force platforms, i.e., solely based on kinematic data combined with a model of the foot-ground contact. The purpose of this work is to provide a more efficient method, using a unilaterally constrained model of the foot at the center of pressure to compute the ground reaction forces. The proposed model does not require any data related with the compliance of the footground contact and is kept as simple as possible. The indeterminacy in the force estimation is handled using a least square approach with filtering. The relative root mean square error (rRMSE) between the numerical estimations and experimental measurements are $4.1 \%$ for the vertical component of the ground reaction forces (GRF), $11.2 \%$ for the anterior component and 5.3\% for the ground reaction moment (GRM) in the sagittal plane.
\end{abstract}

Keywords: Musculoskeletal model, Inverse dynamics, Ground reaction forces, Contact constraint, Rigid foot model

\section{Introduction}

Inverse dynamics simulation based on musculoskeletal models is a common and important method to study and understand the human motion as it can estimate joint torques based on experimental motion analysis data. If the ground reaction forces (GRFs) are directly measured, e.g., using force platforms, this information can be exploited together with the kinematic measurements to estimate the joint torques.

Force platforms can accurately measure the contact reaction along three axes, and provide a good estimation for every type of motion (healthy or pathological gait, run, jump, etc). However, their cost and implementation in the laboratory tend to complicate some studies. A large number of force platforms is necessary to estimate a pathological gait on a long distance, or the race of a sprinter on a specific track. If no force measurement is available, a foot/ground model can be used. Different methods are available in the literature and can be classified in three major categories: empirical, compliant or rigid models.

Empirical models lead to rather simple equations. The GRF can be computed from the measurement of the global center of gravity displacement, as in Farley et al. [10], or statistically as in Keller et al. [15], where data linking the vertical GRF and the walking/running speed are collected.

Compliant models represent the local deformation of the foot sole and the ground during the contact. They are mostly used for predictive models in numerical simulation. Anderson and Pandy [4] and Ackermann and van de Bogert [1] use spring-damper elements to compute the GRF. In Baker and Robb [5], Scott and Winter [23], or Moreira et al. [17], the sole is modelled by deformable spheres in 2D or 3D, giving a precise estimation of the GRF. All these compliant models require an experimental measurement of the compliance between the foot and the ground, whose value might change from one subject to another. The use of compliant models for direct dynamics simulation is thus quite successful but their use in inverse dynamics is hardly achievable because it would require an accurate measurement of the foot sole deformation, which is beyond the capabilities of usual motion capture technologies.

Rigid models neglect the deformation of the foot and the ground and simply rely on a non-penetration condition. Hence, they do not involve any local stiffness and damping parameters, and they do not require any precise evaluation

\footnotetext{
*Corresponding author. Tel.: +32 (0)4 3669113.

Email address: romain.vanhulle@uliege.be (Romain Van Hulle)
} 
of the foot sole deformation. In Koopman et al. [16], or Ren et al. in [20, 21], the GRF are equivalent to the global acceleration of the body, splitted between both feet, according to an empirical law during the double support. The method developed by Oh et al. [18] uses an artificial neural network method for the double support phase. The methods of Fluit et al. [11] or Skals et al. [25] use several contact points per rigid foot and muscle-like actuators to evaluate the GRF through the muscle recruitment optimisation problem. The model proposed by Garcia-Vallejo and Schiehlen [13] uses evolving sets of bilateral constraints in the equation of motion to impose the contact force on the system in direct dynamics simulation.

The purpose of this paper is to provide a general numerical approach to solve the inverse dynamics problem and determine the contact reaction forces, while limiting the number of modelling parameters. The goal is to keep it as simple as possible in 2D, using a rigid body model of the foot in a musculoskeletal model and eluding the muscle recruitment computation and avoiding compliance data. The contact between the foot and the ground is expressed using unilateral contact conditions at the center of pressure, and GRF are modelled using Lagrange multipliers. The internal torques and reaction forces in the joints are represented by Lagrange multipliers associated with the bilateral constraints on the joint angles. The model assumes that the position of the center of pressure is moving as a function of the segment rotation angle in agreement with experimental data. The whole set of joint torques, joint reaction forces and ground reaction forces are obtained by inversion of the equation of motion in a least square sense and a postfiltering method. The results obtained with this method are compared with experimental results obtained with force platforms considered here as the gold standard, and also with other rigid models found in the literature.

\section{Methods}

\subsection{Instrumentation and data acquisition}

The motion was measured by four optoelectronic cameras (Codamotion system; Charnwood Dynamics; Rothley, UK) used to acquire, with a sampling rate of $200 \mathrm{~Hz}$, the position of 28 markers, placed on anatomical points located on the shoulders, hips, knees, ankles, feet and toes.

Two force platforms (Kistler force-plate; Kistler Instrument Corp.; Amherst, NY, USA) were used to obtain reference values of the contact forces and moments for two consecutive steps. The sampling rate of the platforms is 1000 $\mathrm{Hz}$.

Four healthy adults male subjects $(23.8 \pm 2.17$ years old, $177.2 \pm 6.5 \mathrm{~cm}, 74,4 \pm 7,47 \mathrm{~kg})$ completed six gait tests each, for a total of 24 tests. For each test, two consecutive steps were performed on the platforms. The subjects were asked to walk comfortably (mean speed $1.39 \pm 0.19 \mathrm{~m} / \mathrm{s}$ ). The vertical and horizontal GRF, the GRM in the sagital plane and the COP are computed from the force platforms measurement. Two healthy male subjects completed an unilateral squat jump test where they were asked to stand still for two seconds on one foot, then to jump and smoothly land on the same foot.

\subsection{Multi-segment model, generalized coordinates and bilateral constraints}

As shown in Figure 1, the human body is modelled with nine rigid bodies: torso, femur right-left, tibia right-left, foot right-left and toes right-left. These bodies evolve in the sagital plane and are represented by three control points: the proximal and distal extremities and the center of mass. The position of these centers of mass relatively to the extremities comes from anthropometric tables [26].

To describe the motion of a multibody system, relative or absolute coordinates can be chosen [14]. For biomechanical problems, relative independent coordinates (e.g. joints angles and pelvis position and orientation) are usually used, and no bilateral constraint needs to be considered. In this paper, absolute coordinates are chosen: compared to relative coordinate formulations, this approach leads to simpler equations of motion and a straightforward evaluation of the reactions forces in the bodies and joints, which are represented by the Lagrange multipliers. The following generalized coordinates (g.c.) $\mathbf{q}(t)$ are chosen: the $x$ and $y$ coordinates of the extremities and of the center of mass of each segment (42 g.c), the orientation $\theta$ (angle between the segment and the ground) of each segment (9 g.c) and the relative angle between two consecutive segments are also taken into account $(8 \mathrm{~g} . \mathrm{c})$. The vector of generalized coordinates contains 59 components. The displacements of segment extremities in the sagittal plane, labelled with I...XII in Figure 1 are measured experimentally. Hence, the fitting of these generalized coordinates on the measured marker trajectories is a trivial problem, which is an additional advantage of using an absolute coordinates model. The other coordinates are then calculated based on kinematic considerations to complete the vector. As the out-of-plane motion is ignored, the vector of generalized coordinates may slightly violate the rigidity constraints. In this study, this artifact leads to a variation of link lengths of about $1 \%$, which can be neglected for the purpose of an inverse dynamics analysis. In case 


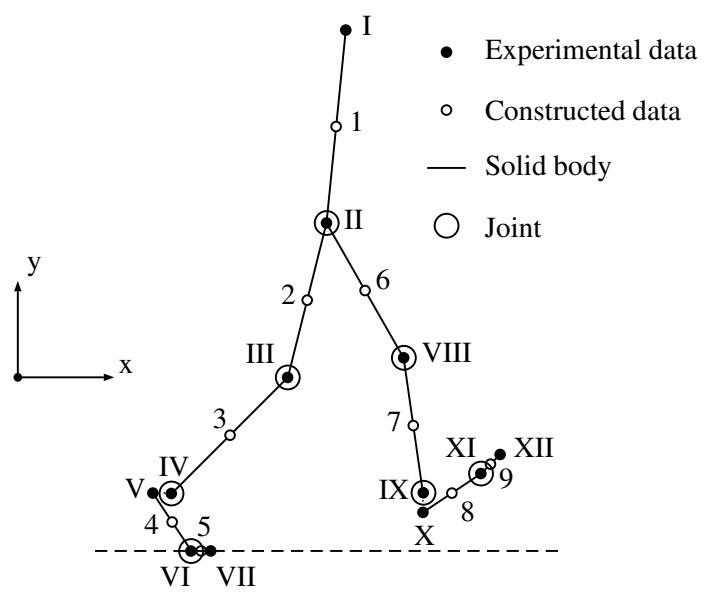

Figure 1: Muskuloskeletal model

of larger out-of-plane motions, an optimization procedure could be used to ensure the consistency, as in [3]. As the model has 11 degrees of freedom and 59 generalized coordinates, 48 scleronomic bilateral constraints are introduced and represent the rigid body conditions and the constraints defining the joint angles from the absolute coordinates. In the inverse dynamics analysis, the relative joint angles are piloted by the experimental data, leading to eight additional rheonomic bilateral constraints.

The set of 56 bilateral constraints is expressed as:

$$
\mathbf{g}^{\mathcal{B}}(\mathbf{q}, t)=\mathbf{0}
$$

As the joint angles are prescribed, three degrees of freedom remain, namely the vertical and lateral motion of the global center of gravity and its orientation.

The velocity vector $\dot{\mathbf{q}}(t)$ and the acceleration vector $\ddot{\mathbf{q}}(t)$ are computed to be consistent with respect to the bilateral constraints. To avoid a coupling between the computation of the velocities and accelerations and the algorithm for the activation of the contact constraints (Section 2.5), the contact constraints are disregarded at this level, following a similar strategy as in [26]. First, the velocity and acceleration of a subset of independent coordinates (joints angles and pelvis position and orientation) are obtained through numerical time differentiation, based on central difference scheme and filtered using a Butterworth fourth-order zero-shift low-pass filter, with a cut-off frequency of $\omega_{\text {kin }}$, set to $6 \mathrm{~Hz}$ in accordance with the literature [26]. The velocity and acceleration of the dependent coordinates are then reconstructed by solving the bilateral constraints at velocity and acceleration levels to be consistent with the rigid body assumption [14].

\subsection{Model of the foot and contact constraints}

As shown in Figure 2, the displacements of each foot are measured by four optoelectronic markers: on the heel, on the malleolus (representing the joint between the foot and the tibia), on the head of the first metatarsal bone (joint between the foot and the toes), and on the hallux. However, these markers never touch the ground, and moreover, depending on the orientation of the foot, the distance between the ground and those markers when contact occurs may change. To obtain a simple expression of the foot/ground distance, three new control points on the foot ( $A, B$ and $C$ ) are placed at the ground level and at the vertical of the experimental markers during a preliminary static test. These three points are rigidly connected to the points $V, V I$ and $V I I$ respectively, and follow the motion of the foot segments.

At this level, a first model of the foot is proposed by imposing a non penetration and a non-slipping condition at points $A, B$ and $C$. However, this approach leads to irregularities and jumps of the ground reaction moment (GRM) and of the position of the center of pressure because of the coarse spatial localization of the center of pressure (COP), as will be shown in the results and in Figure 3.

To prevent these irregularities, a second model of the foot is proposed in this paper. Based on the three control points defined previously $(A, B$ and $C$ ), one contact point $D$ moving from $A$ to $C$ and representing the COP is created. Experimentally, we observe that $x_{D}$ is strongly correlated with the foot segment inclination $\theta_{4}$. Figure 3 shows the mean value of $x_{D}$ as a function of $\theta_{4}$ obtained from the force platforms measurements for gait and squat jump tests. 


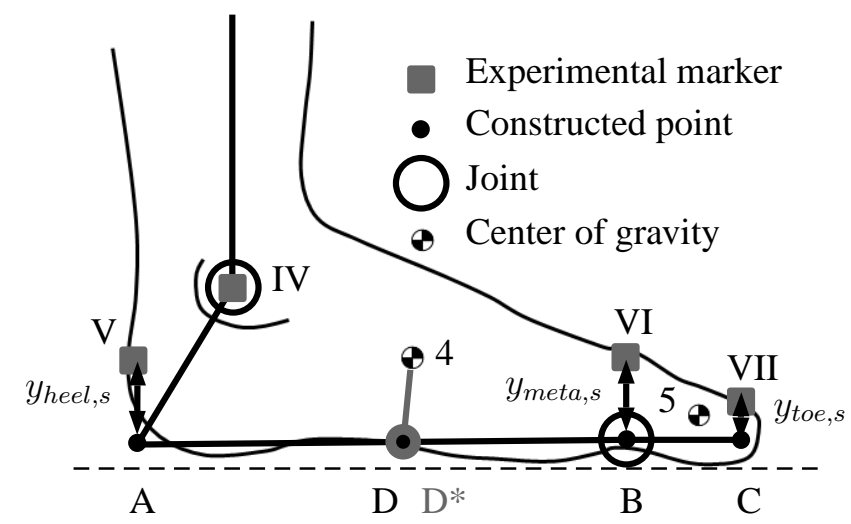

Figure 2: Model of the left foot

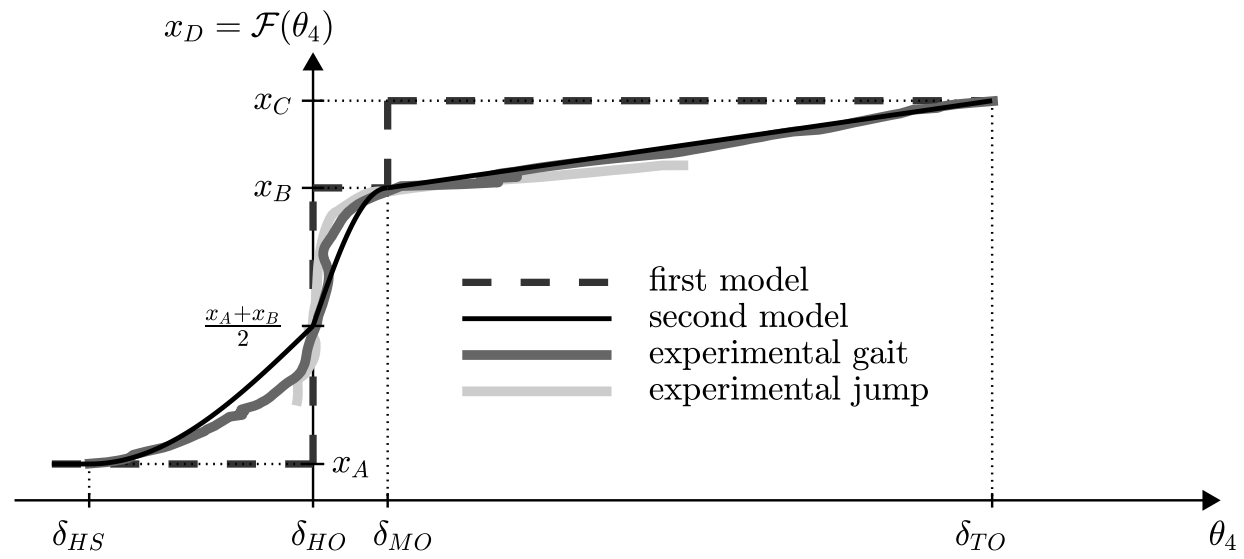

Figure 3: Evolution of the position of the COP

Based on the experimental data, the position of the COP $x_{D}$ as a function of $\theta_{4}$ seems to be independent of the activity or subject. Hence, we assume that the position of the contact point $D$ is a function of $y_{A}, y_{B}, y_{C}$ and $\theta_{4}$ as follows:

$$
\begin{aligned}
& y_{D}=\min \left(y_{A}, y_{B}, y_{C}\right) \\
& x_{D}=\mathcal{F}\left(\theta_{4}\right)
\end{aligned}
$$

where the function $\mathcal{F}$ is defined to fit mean experimental data. In this paper, we propose a function defined in three parts between major gait events: a first sinusoidal function between the heel strike $\left(\theta_{4}=\delta_{H S}\right)$ and the heel off $\left(\theta_{4}=\delta_{H O}\right)$, a second sinusoidal function between the heel off and the metatarsal off $\left(\theta_{4}=\delta_{M O}\right)$, and a linear function between the metatarsal off and the toe off $\left(\theta_{4}=\delta_{T O}\right)$. The function is thus fully determined by the four parameters $\delta_{H S}, \delta_{H O}, \delta_{M O}$ and $\delta_{T O}$.

As $D$ is moving with respect to the foot, we then define the point $D^{*}$ which instantaneously coincides with point $D$ and is rigidly fixed to the foot. The four coordinates $x_{D^{*}}$ and $y_{D^{*}}$ of the two feet are thus added to the vector of generalized coordinates $\mathbf{q}(t)$, which contains now 63 components. Also, four new bilateral constraints are added to represent the rigid connexion between the $D^{*}$ and the points 4 and 8 of the two feet.

Finally, the GRF are computed by using a unilateral normal constraint and a tangential sticking constraint at the point $D^{*}$, see also the rolling without slipping tangential condition in Caspers et al. [9]. First, the normal nonpenetration condition takes the form of a complementarity condition between the normal gap distance $\mathrm{g}^{*}, n$ and the normal force $\lambda^{D^{*}, n}$ :

$$
\mathrm{g}^{D^{*}, n}(\mathbf{q})=y_{D}^{*} \geq 0, \quad \lambda^{D^{*}, n} \geq 0, \quad \mathrm{~g}^{D^{*}, n}(\mathbf{q}) \lambda^{D^{*}, n}=0
$$


In the inverse dynamics analysis proposed in this paper, these conditions are not explicitly imposed but they are exploited to build the algorithm for the generalized forces, as explained below.

In the tangent direction, the contact condition can be represented by a Coulomb friction model with a distinction between stick and slip situations. In this study, we assume that the foot does not slip on the ground so that all closed contact are in stick state [19]. This means that the tangent displacement $\mathrm{g}^{D^{*}, t}$ and the tangent reaction forces $\lambda^{D^{*}, t}$ satisfy:

$$
\begin{array}{rc}
\text { if } & \mathrm{g} D^{*}, n \\
\text { else } & \lambda^{D^{*}, t}=0
\end{array}
$$

The normal GRF (resp. tangent) is given by the Lagrange multipliers $\lambda^{D^{*}, n}$ and $\lambda^{D^{*}, t}$ of the normal (resp. tangent) constraints, for each foot. The center of pressure (COP) of the foot is given by $x_{D}$.

The total set of bilateral constraints and contact constraints (such as Eq. (3)-(4)) are collected in the vector $\mathbf{g}(\mathbf{q}, t)$.

\subsection{Dynamic equilibrium and least square evaluation of the forces}

The dynamic equilibrium of the mechanical system is given by 63 equations, written in the following matrix form $[2,8,24]$ :

$$
\mathbf{M} \ddot{\mathbf{q}}+\mathbf{g}_{\mathbf{q}}^{\mathrm{T}}(\mathbf{q}, t) \boldsymbol{\lambda}-\mathbf{f}=\mathbf{0}
$$

where the mass matrix $\mathbf{M}$ is constructed thanks to anthropometric tables [26], $\mathbf{g}_{\mathbf{q}}(\mathbf{q}, t)=\frac{\partial \mathbf{g}(\mathbf{q}, t)}{\partial \mathbf{q}}$ is the matrix of bilateral and unilateral constraints gradients, $\boldsymbol{\lambda}$ is the vector of bilateral and unilateral Lagrange multipliers, and $\mathbf{f}$ represent the gravity acting on the system. Equation (5) represents the balance between the inertial forces, the internal forces resulting from the constraints $\mathbf{g}_{\mathbf{q}}^{\mathrm{T}} \boldsymbol{\lambda}$ and the external forces.

In the inverse dynamics problem, the accelerations $\ddot{\mathbf{q}}$ are given and the Lagrange multipliers constitute the sole unknowns. For a given $\ddot{\mathbf{q}}$, De Jalon and Gutiérrez-Lopez [12] show that the global resultant of the forces $\mathbf{g}_{\mathbf{q}}^{\mathrm{T}} \boldsymbol{\lambda}$ is unique but that the vector of Lagrange multipliers $\boldsymbol{\lambda}$ remains indeterminate if the matrix $\mathbf{g}_{\mathbf{q}}^{\mathrm{T}}$ does not have maximum rank, in other words, if the constraints are redundant.

To obtain the unknowns $\boldsymbol{\lambda}$, we develop Equation (5), and use Equations (1) to (4) to define the set of active constraints. In Equation (5), it is essential to separate the set of active constraints $\mathcal{A}$, whose Lagrange multipliers can differ from zero from inactive ones $\overline{\mathcal{A}}$, whose Lagrange multipliers are zero. The classification criterion will be detailed in Section 2.5. We obtain:

$$
\mathbf{g}_{\mathbf{q}}^{\mathcal{A}, \mathrm{T}}(\mathbf{q}) \lambda^{\mathcal{A}}=-\mathbf{M} \ddot{\mathbf{q}}+\mathbf{f}, \quad \lambda^{\overline{\mathcal{A}}}=\mathbf{0}
$$

Depending on the number of active constraints in the system and their degree of redundancy, the problem might be over- and/or underdeterminated and the solution for the Lagrange multipliers $\lambda$ might not be unique. However, at the global level, the resultant $\mathbf{g}_{\mathbf{q}}^{\mathcal{A}, \mathrm{T}}(\mathbf{q}) \boldsymbol{\lambda}^{\mathcal{A}}$ is unique and has a physical meaning. We postulate that usual overground motions tend to minimize the full set of reaction forces. Hence, the over/underdetermination is handled using the least square method and the pseudo-inverse [6, 12]:

$$
\lambda^{\mathcal{A}}=\left(\mathbf{g}_{\mathbf{q}}^{\mathcal{A}, \mathrm{T}}(\mathbf{q})\right)^{+}(\mathbf{f}-\mathbf{M} \ddot{\mathbf{q}}), \quad \lambda^{\overline{\mathcal{A}}}=\mathbf{0}
$$

To eliminate the possible indeterminacy, other optimisations could be performed: e.g. the minimisation of the metabolic energy consumption. It would lead to another weighting of the constraints, or even to another equation to solve. According to our experience, minimizing the full set of reaction forces in a least-square sense leads to satisfactory results, as will be seen in Section 3.

\subsection{Contact activation criterion}

The contact activation criterion is based on the complementarity condition, Equation (3), between the gap function $\mathrm{g}^{j, n}$ and the normal reaction force $\lambda^{j, n}$. In our context, $\mathrm{g}^{j, n}$ is evaluated from kinematic measurements and $\lambda^{j, n}$ is estimated by our inverse dynamics scheme. Hence, both variables are affected by some uncertainties, and a robust classification criterion is needed. Inspired by the work of Alart and Curnier [2], a mixed criterion relying on both values of $\mathrm{g}^{j, n}$ and $\lambda^{j, n}$ is proposed so that the set of active contacts $\mathcal{C}$ is defined as (Figure 4):

$$
\mathcal{C}=\left\{j: \lambda^{j, n}-\mathrm{rg}^{j, n} \geq 0\right\}
$$




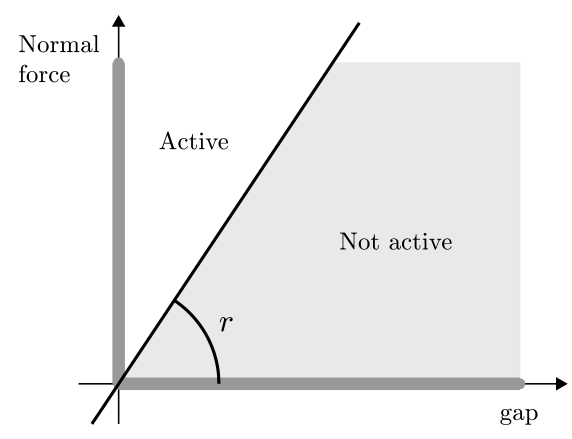

Figure 4: Complementarity condition (bold grey) and mixed constraint activation criterion, [2]

where $r \geq 0$ is a scalar parameter whose chosen value will be discussed in Section 4 . We also define the set of inactive contacts:

$$
\bar{C}=\left\{j: \lambda^{j, n}-r \mathrm{~g}^{j, n}<0\right\}
$$

For every contact $j$ in $\mathcal{C}$, the constraints $\mathrm{g}^{j, n}=\mathrm{g}^{j, t}=0$, whereas for all contacts $j$ in $\overline{\mathcal{C}}$, the Lagrange multipliers are fixed to zero $\lambda^{j, n}=\lambda^{j, t}=0$. These conditions are thus fully equivalent to Equations (3) and (4). At every time, we thus impose:

$$
\mathbf{g}^{\mathcal{A}}=\left[\begin{array}{c}
\mathbf{g}^{\mathcal{B}} \\
\mathbf{g}^{\mathcal{C}, n} \\
\mathbf{g}^{\mathcal{C}, t}
\end{array}\right]=0 \quad \text { and } \quad \lambda^{\overline{\mathcal{A}}}=\left[\begin{array}{l}
\lambda^{\overline{\mathcal{C}}, n} \\
\lambda^{\overline{\mathcal{C}}, t}
\end{array}\right]=0
$$

where the sets $\mathcal{C}, \mathcal{A}$ and $\overline{\mathcal{A}}$ implicitly depend on $\mathbf{q}$ and $\boldsymbol{\lambda}$ (see Equations (8), (9) and (10)).

Combining the activation criterion with Equation (6) leads to an implicit problem for $\boldsymbol{\lambda}$ which can be solved numerically. Here, an iterative Newton semi-smooth solver is selected (Figure 5). The process is initialized by considering that every contact constraint is active. Then, Equation (7) is used to evaluate a first guess of the Lagrange multipliers. The activation status is then reevaluated using these Lagrange multipliers and the activation criterion in Equations (8) and (9). A new distribution of the Lagrange multipliers is determined in the next iteration, and the set of active contact constraints is again updated, until the proper contact status of each constraint is found.

\subsection{Final filtering}

The least-square solution might exhibit sudden and abrupt changes from one sampling time to the next, especially when activating and deactivating contact constraints. To ensure the temporal continuity of the GRF, a post-process final filtering of the results is applied to the previous results, using a Butterworth fourth-order zero-shift low pass filter, with a cut-off frequency $\omega_{d y n}$, whose chosen value will also be discussed in Section 4 . After filtering, $\lambda^{\overline{\mathcal{A}}}$ is reset to zero to ensure the consistency with the activation status.

\section{Results}

The measurements on the force platforms are used as the reference results, and the errors in the numerically computed reaction forces are based on the Root Mean Square Error (RMSE):

$$
\mathrm{RMSE}=\sqrt{\frac{1}{T} \int_{0}^{T}\left(u_{\text {num }}(t)-u_{\text {exp }}(t)\right)^{2} d t}
$$

where $T$ is the duration of the test, limited to two steps, $u_{\text {num }}(t)$ and $u_{\text {exp }}(t)$ are respectively the numerical estimation and the experimental measurement of the GRF/GRM. The GRM are computed relatively to a point located between the force platforms. 


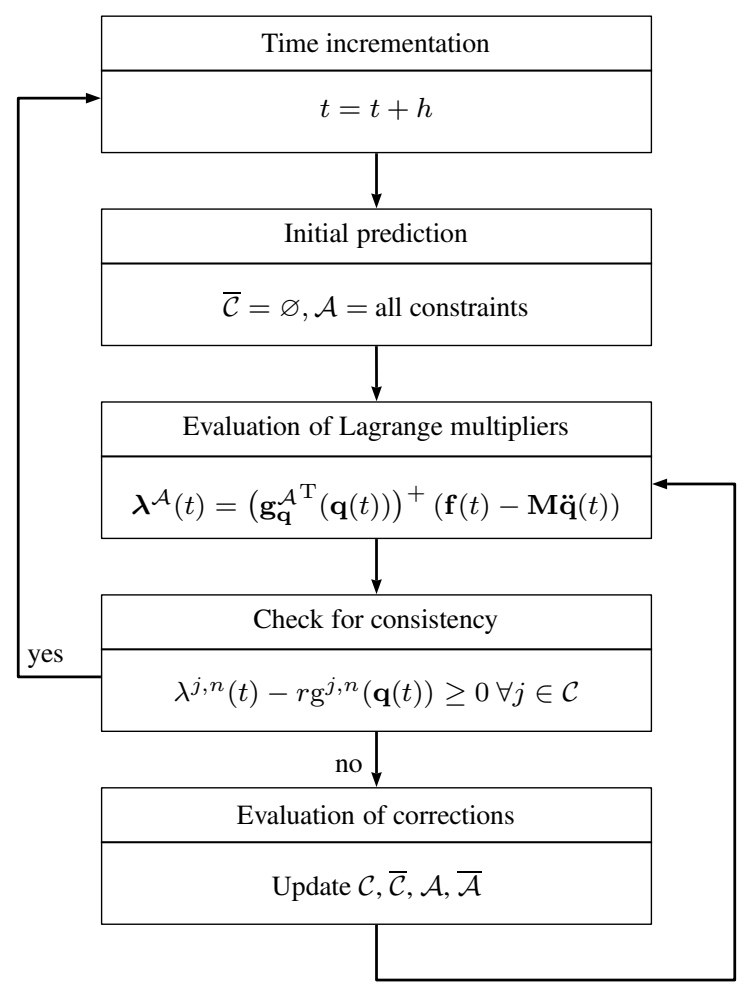

Figure 5: Evaluation of the force using a Newton semi-smooth solver

Also, the relative Root Mean Square Error (rRMSE) is defined as [18]:

$$
\mathrm{rRMSE}=\frac{100 \text { RMSE }}{\frac{1}{2}\left[\max \left(u_{\text {num }}(t)\right)-\min \left(u_{\text {num }}(t)\right)+\max \left(u_{\text {exp }}(t)\right)-\min \left(u_{\text {exp }}(t)\right)\right]}
$$

Finally, in this study, the numerical value of the parameters are set to $\delta_{H S}=-0.3 \mathrm{rad}, \delta_{H O}=0 \mathrm{rad}, \delta_{M O}=0.15 \mathrm{rad}$, $\delta_{T O}=1 \mathrm{rad}$, based on the mean value obtained experimentally for the 24 gait tests and consistent with the literature [7], $r=10^{3} \mathrm{~N} / \mathrm{m}, \omega_{\text {kin }}=6 \mathrm{~Hz}$ and $\omega_{d y n}=12 \mathrm{~Hz}$.

\subsection{Healthy gait tests}

In Figure 6, the results are illustrated for one single gait test, with the two proposed foot models.

For the 24 tests, with the single point foot model, the rRMSE and standard deviation (SD) of the vertical GRF are 4.1\%(1.1). The rRMSE and (SD) of the horizontal GRF are 11.2\%(1.7). The rRMSE and (SD) of the GRM are only $5.3 \%(1.1)$, which is similar to the error on the vertical GRF.

\subsection{Jump tests}

The method, coupled with the second foot model, is tested for a unilateral squat jump test and the results are shown in Figure 7. The rRMSE and (SD) are smaller for the vertical GRF, 2.4\%(0.4), compared to the horizontal GRF, $5.6 \%(0.6)$. It comes from the fact that there is almost no movement on the horizontal direction, and mainly noise is measured. The rRMSE and (SD) of the GRM are 5\%(0.4).

\section{Discussion}

A method is developed for the inverse dynamics analysis of the human motion, based on muskuloskeletal models using rigid segments and a unilaterally constrained foot-ground contact model. The proposed method provides results with limited errors. It relies on two main assumptions: the position of the COP is assumed to be a function of the segment angle and the full set of reaction forces is evaluated in a least-square sense. The parameters are the activation parameter $r$, the cut-off frequencies $\omega_{k i n}$ and $\omega_{d y n}$ of the filters, and the limit angles $\delta$. 

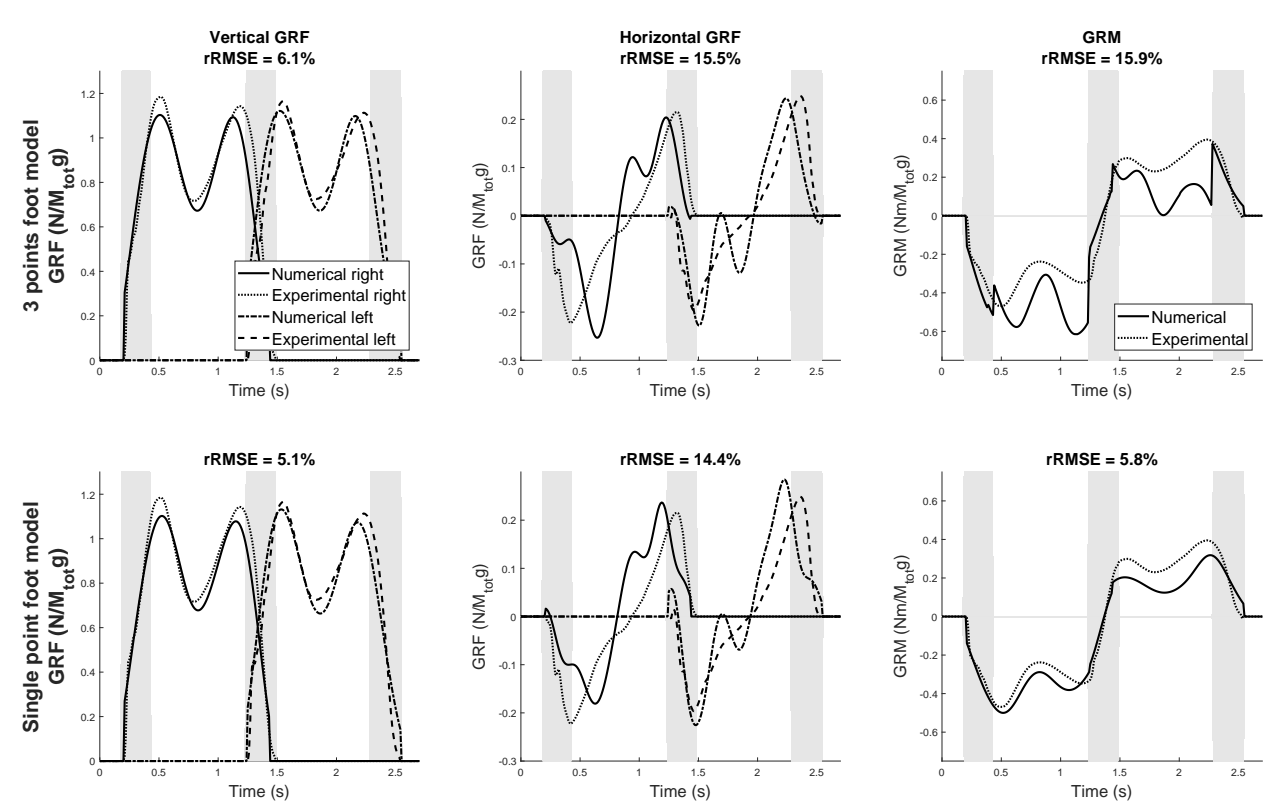

Figure 6: Comparison of the 3-points foot model and single point foot model with experimental measurements for one single gait test.
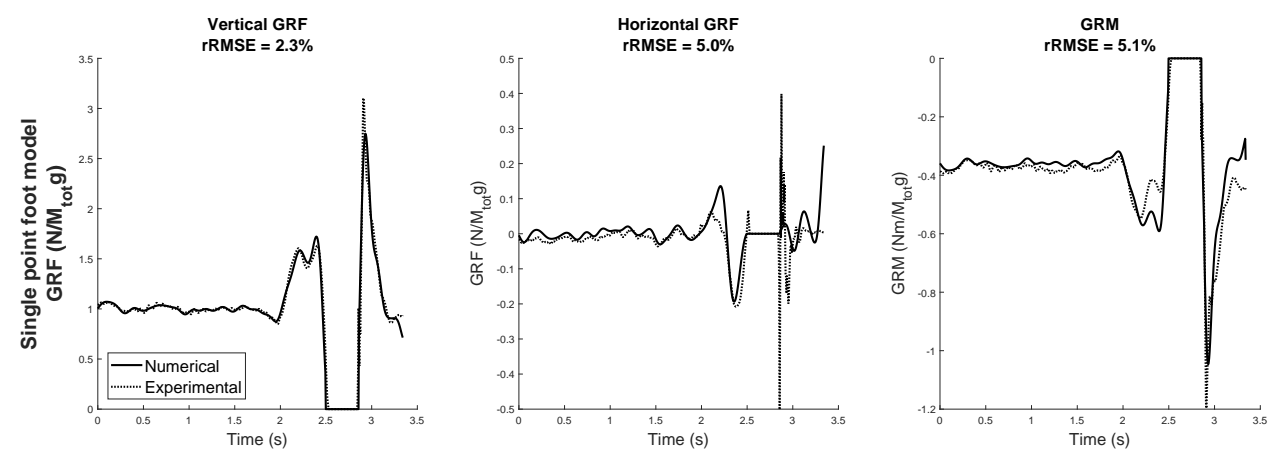

Figure 7: Comparison of the single point foot model numerical results with experimental measurement for one single squat jump test. 
In the second model, the assumption that the COP evolves as a continuous function $\mathcal{F}$ of the segment angle is relevant for a variety of human movements (e.g. healthy gait, run, stance, jump,...). This assumption mostly affects the COP position (and thus the GRM), but a very limited influence on the value of the contact forces. It leads to a clear improvement of the GRM between the first and second model. In this paper, the function $\mathcal{F}$ is defined by parts based on gait events (heel strike, heel off, metatarsal off and toe off) to fit properly the mean experimental data obtained during the gait and jump tests performed for this study. This assumption is clearly an approximation but our results confirm its relevance. The parameters $\delta$ of the function $\mathcal{F}$ have a clear biomechanical interpretation and have been measured and averaged for the 24 gait tests, leading to consistent values with the literature. Using a subject specific value of these parameters could further improve the results. However, in our experience, the results are quite robust with respect to these parameters and even to the choice of the function $\mathcal{F}$. In the case of high speed running or pathological gait, the motion of the COP can be more complex and a new COP function may thus be required (averaged or specific).

The model postulates a least-square distribution of the reaction forces, i.e., it assumes that during motion, the human body tends to minimize the value of those forces. This assumption is used to resolve the indeterminacy in the distribution of the horizontal GRF over the two feet which is particularly critical during the double support phase. It seems reasonable for many day-to-day activities and is confirmed by our results for gait and squat jump tests. When high performance is pursued or with some pathological behaviours, this assumption should be reconsidered on a case-by-case basis. Other cost functions could be used to eliminate the indeterminacy of the solution, such as the minimisation of the metabolic energy, for example.

The activation parameter $r$ can be selected to obtain robust results with respect to the measurement of the error on the displacement of the foot. With a small $r$, the activation criterion is more sensitive to the errors on the force estimation. Conversely, with a large $r$, the criterion is more sensitive to the errors in the gap measurement. If $r=0$, it only relies on the repulsive computed forces and if $r=+\infty$, it is only influenced by the kinematics. As the errors on the kinematic measurements are usually quite small, a large value of $r$ is recommended: in our experience with standard gait and jump tests, the criterion is robust and leads to equivalent results for any $r>10 \mathrm{~N} / \mathrm{m}$. Lower values, $r<10 \mathrm{~N} / \mathrm{m}$, lead to over-activation of the constraints. To test the robustness of this criterion with respect to uncertainties in the gap distance measurement, the ground level is virtually shifted up or down by $1 \mathrm{~cm}$, which may represent an error in the calibration of the motion capture devices or irregularities in the ground plane. The value of $r$ had to be chosen in the smaller interval $\left[10^{2}, 10^{4}\right] \mathrm{N} / \mathrm{m}$ to obtain acceptable results. In particular, values lower than $10^{2} \mathrm{~N} / \mathrm{m}$ create the same issues than in the undisturbed case, and values higher than $10^{4} \mathrm{~N} / \mathrm{m}$ lead to an over/under-activation of the constraints.

The kinematic data (velocities and accelerations) are computed using a Butterworth $4^{\text {th }}$ order low-pass filter with a cut-off frequency $\omega_{\text {kin }}$ set to $6 \mathrm{~Hz}$, as usually recommended [26]. The cut-off frequency $\omega_{d y n}$ of the post-filtering process is used to ensure the temporal continuity of the GRF, especially during the activation and deactivation of the contact constraints (e.g. the transition between single and double support). With a low cut-off frequency, the loss of information might become significant and the value of the GRF non-physical. With a high value, discontinuities appear in the GRFs, leading to larger errors. In this study, it was selected as twice the value of $\omega_{k i n}$, hence $\omega_{d y n}=12 \mathrm{~Hz}$.

In Table 1, the proposed method is compared with the methods of Ren et al. [21], of Oh et al. [18] and of Fluit et al. [11] for the healthy gait test. One observes that the proposed method provides comparable results for the vertical GRF and the GRM. The main advantage of the proposed method is in the simplicity of the foot/ground contact model which only involves a limited number of parameters which do not necessarily need to be adapted to the specific subject or task under study. Several assumptions have been made: the planar motion, no slipping conditions, ideal distribution of masses and inertia (according to anthropometric tables), absence of friction in the simplified articular joints, unilateral contact between the foot and the ground, and simple function of the COP evolution. Despite these assumptions, the robustness of the model and the activation criterion makes it possible to obtain valuable results.

\section{Conclusion}

A method is proposed to compute the GRF and GRM for healthy gait tests and jumps based on a least square estimation of the Lagrange multipliers of a unilaterally constrained foot model. A mixed criterion is used for a robust and consistent activation of the contact constraints and a simple model of the foot serves to evaluate the position of the center of pressure.

This method produces reliable results. Easy to implement, it could be used to estimate the GRF and GRM in many cases: healthy and pathological gait, jumping, running, treadmill, etc. In future works, the model will be improved in order to obtain exploitable results for the GRF along the lateral axis as well as the GRM in the transverse and frontal planes during various types of $3 \mathrm{D}$ gait and human activities. 


\begin{tabular}{lllll}
\hline Method & $\begin{array}{l}\text { Smooth transi- } \\
\text { tion assumption } \\
\text { method (Ren et } \\
\text { al., [22]) }\end{array}$ & $\begin{array}{l}\text { Hybrid } \\
\text { kinematics- } \\
\text { neural method } \\
\text { (Oh et al., [18]) }\end{array}$ & $\begin{array}{l}\text { Muscle-like ac- } \\
\text { tuators method } \\
\text { (Fluit et al., [11]) }\end{array}$ & $\begin{array}{l}\text { Proposed nons- } \\
\text { mooth method }\end{array}$ \\
Participants & $N=5$ & $N=48$ & $N=9$ & $N=4$ \\
\hline Axes/Planes & rRMSE (SD) & rRMSE (SD) & rRMSE (SD) & rRMSE (SD) \\
\hline Vertical & $6.5(1.3)$ & $5.8(1.0)$ & $2.7(0.6)$ & $4.1(1.1)$ \\
Anterior & $10.5(0.8)$ & $7.3(0.8)$ & $9.3(1.2)$ & $11.2(1.7)$ \\
Sagittal & $11.7(3.4)$ & $9.9(1.9)$ & $7.4(1.8)$ & $5.3(1.1)$ \\
\hline
\end{tabular}

Table 1: Gait test: results comparison

\section{Conflict of interest statement}

We confirm that there are no conflicts of interest associated with this publication.

[1] Ackermann M. and van de Bogert A.J., 2010. Optimality principles for model-based prediction of human gait. Journal of Biomechanics 43, 1055-1060.

[2] Alart P. and Curnier A., 1991. A mixed formulation for frictional contact problems to Newton like solution. Computer Methods in Applied Mechanics and Engineering 92, 353-375.

[3] Alonso F.J., Cuadrado J., Lugrís U. and Pintado P., 2010. A compact smoothing-differentiation and projection approach for the kinematic data consistency of biomechanical systems. Multibody System Dynamics 24, 67-80.

[4] Anderson F.C. and Pandy M.G., 2001. Dynamic optimization of human walking. Journal of Biomechanichal Engineering 123, 381-390.

[5] Baker R. and Robb J., 2006. Foot models for clinical gait analysis. Gait and posture 23, 399-400.

[6] Blumentals A., Brogliato B. and Bertails-Descoubes F., 2016. The contact problem in Lagrangian systems subject to bilateral and unilateral constraints, with or without sliding Coulomb's friction: a tutorial. Multibody System Dynamics 38, 43-76.

[7] Borghese N.A., Bianchi L., Lacquaniti F., 1996. Kinematic determinants of human locomotion. Journal of Physiology 494(3), 863-879.

[8] Brogliato B., 1999. Nonsmooth Mechanics. Springer-Verlag, London.

[9] Caspers L., Siebler M., Hefter H., Lugrís U. and Kecskeméthy A., 2017. Using kinematic rolling surfaces for fast foot-ground modeling in the forward dynamics of human gait - A sagittal plane analysis. ECCOMAS Thematic Conference on Multibody Dynamics.

[10] Farley C.T. and Gonzales O., 1996. Leg stiffness and stride frequency in human running. Journal of Biomechanics 29, 181-186.

[11] Fluit R., Andersen M.S., Nolk S., Verdonschot N. and Koopman H.F.J.M., 2014. Prediction of ground reaction forces and moments during various activities of daily living. Journal of Biomechanics 47, 2321-2329.

[12] García de Jalón J. and Gutiérrez-Lopez M.D., 2013. Multibody dynamics with redundant constraints and singular mass matrix: existence, uniqueness, and determination of solutions for accelerations and constraint forces. Multibody System Dynamics 30, 311-341.

[13] Garcia-Vallejo D. and Schiehlen W., 2012. 3D-simulation of human walking by parameter optimization. Archive of Applied Mechanics 82, 533-556. 
[14] Géradin M. and Cardona A., 2001. Flexible Multibody Dynamics: A finite Element Approach. John Wiley \& Sons Ltd, Chichester.

[15] Keller T.S., Weisberger A.M., Ray J.L., Hasan S.S. and Shiavi R.G., 1996. Relationship between vertical ground reaction force and speed during walking, slow jogging, and running. Clinical Biomechanics 11, 253-259.

[16] Koopman B., Grootenboer H.J. and de Jongh H.J., 1995. An inverse dynamics model for the analysis, reconstruction and prediction of bipedal walking. Journal of Biomechanics 28, 1369-1376.

[17] Moreira P., Silva M. and Flores P., 2009. Ground foot interaction in human gait, modelling and simulation. Euromech Solid Mechanics Conference.

[18] Oh S.E., Choi A. and Mun J.H., 2013. Prediction of ground reaction forces during gait based on kinematics and a neural network model. Journal of Biomechanics 46, 2372-2380.

[19] Pàmies-Vilà R., Gonzalez F., Kövecses J. and Font-Llagunes J.M., 2018. Use of performance indicators in the analysis of running gait impacts. Multibody System Dynamics 43, 131-151.

[20] Ren L., Jones R.K. and Howard D., 2005. Dynamic analysis of load carriage biomechanics during level walking. Journal of Biomechanics 38, 853-863.

[21] Ren L., Jones R.K. and Howard D., 2007. Predictive modelling of human walking over a complete gait cycle. Journal of Biomechanics 40, 1567-1574.

[22] Ren L., Jones R.K. and Howard D., 2008. Whole body inverse dynamics over a complete gait cycle based only on measured kinematics. Journal of Biomechanics 41, 2750-2759.

[23] Scott S.H. and Winter D.A., 1993. Biomechanical model of the human foot: Kinematics and kinetics during the stance phase of walking. Journal of Biomechanics 26, 1091-1104.

[24] Silva M.P.T. and Ambrosio J.A.C., 2002. Kinematic data consistency in the inverse dynamic analysis of biomechanical systems. Multibody System Dynamics 8, 219-239.

[25] Skals S., Ki Jung M., Damsgaard M. and Andersen M.S., 2016. Prediction of ground reaction forces and moments during sport-related movements. Multibody System Dynamics 39, 175-195.

[26] Winter D.A., 2009. Biomechanics and Motor Control of Human Movement, fourth ed. John Wiley \& Sons, Inc., Hoboken, New Jersey. 